\title{
RAB40C Gene Polymorphisms Were Associated with Alcohol-Induced Osteonecrosis of the Femoral
} Head

Chang Liu',*

Xuan $\mathrm{Liu}^{2, *}$

Xiaowei $\mathrm{Li}^{2}$

'Department of Emergency, The Second Hospital of Tangshan, Tangshan, Hebei Province, 063000, People's Republic of China; ${ }^{2}$ Department of Orthopedics, The Hospital of Yutian County, Tangshan, Hebei Province, 063000, People's Republic of China

*These authors contributed equally to this work
Correspondence: Chang Liu Department of Emergency, The Second Hospital of Tangshan, Tangshan, Hebei Province, 063000, People's Republic of China

Tel/Fax +86- 18633328305

Email liuchang991204@I63.com
Introduction: Alcohol-induced osteonecrosis of the femoral head (ONFH), a progressive disease, is caused by excessive drinking and genetic factors. Currently, it remains to represent a significant challenge. The association between alcohol-induced ONFH and $R A B 40 C$ gene polymorphisms may provide a direction for the mechanism of alcoholic ONFH.

Methods: A total of 201 alcohol-induced ONFH patients and 201 healthy controls were recruited in this case-control study. The polymorphisms of $R A B 40 C$ gene were genotyped in blood samples by Agena MassARRAY RS1000. Pearson chi-square test was used to calculate difference in allele frequencies of gene polymorphisms between the cases and controls. Alcohol-induced ONFH risk was estimated using odds ratios (ORs) and 95\% confidence intervals (CIs).

Results: In the overall analysis, the allele "G" of rs62030917 was significantly increased alcohol-induced ONFH risk $(\mathrm{OR}=1.47,95 \% \mathrm{CI}=1.07-2.02, p=0.017)$ in the allele model. In the genetic analysis, rs62030917 also increased the risk of alcohol-induced ONFH in the dominant model (adjusted $\mathrm{OR}=1.52,95 \% \mathrm{CI}=1.02-2.26, p=0.039$ ) and the log-additive model (adjusted $\mathrm{OR}=1.42,95 \% \mathrm{CI}=1.05-1.93, p=0.025$ ). Age stratification analysis suggested that rs62030917 increased the risk of alcohol-induced ONFH among the individuals younger than 42 years old. Moreover, carriers of AA, GA and GG genotypes in rs2269556 had LDL-C levels that were significantly different ( $p=0.047)$. Among them, carriers of GG genotype had the highest LDL-C levels.

Conclusion: This study revealed rs62030917 in $R A B 40 C$ gene might increase the risk of alcohol-induced ONFH, providing a theoretical basis for the mechanism of $R A B 40 C$ in alcohol-induced ONFH.

Keywords: alcohol-induced ONFH, RAB40C, single nucleotide polymorphisms

\section{Introduction}

Osteonecrosis of the femoral head (ONFH) is a disease in which the local death of osteocytes and bone marrow components is caused by venous congestion or impaired arterial blood supply or the femoral head rupture. ${ }^{1,2}$ ONFH usually occurs between 30 and 50 years old. ${ }^{1}$ It is reported that the risk factors of ONFH included corticosteroid use, trauma, alcohol consumption, coagulation abnormalities, hyperlipidemia, and smoking. ${ }^{1}$ Numerous epidemiological and multicenter studies have shown that most ONFH patients have a history of alcohol abuse. It has been reported that alcohol can destroy bone homeostasis by directly 
inhibiting the proliferation and differentiation of bone marrow mesenchymal stem cells (BMSCs). ${ }^{3}$ In particularly, alcohol significantly inhibited the proliferation and DNA synthesis of osteoprogenitor cells. ${ }^{4}$ Therefore, alcohol is a factor that cannot be ignored to cause osteonecrosis of the femoral head. ${ }^{5}$ At present, there are two main types of osteonecrosis: traumatic and non-traumatic. ${ }^{2}$ Alcohol-induced ONFH belongs to non-traumatic osteonecrosis. ${ }^{6}$ Epidemiological studies have reported that $20-45 \%$ of ONFH patients are associated with alcohol consumption. ${ }^{1}$ Excessive drinking may lead to dyslipidemia, abnormal differentiation of BMSCs differentiation, and abnormal bone metabolism. In addition, alcohol has a significant dose effect on bone homeostasis. ${ }^{7}$ However, each individual have different susceptibility, which may be related to genetic predispositions. ${ }^{8}$ Some studied showed that $M M P-8$ and $M M P-3$ gene polymorphisms were related to the risk of alcohol-induced ONFH. ${ }^{9}$

$R A B 40 C$, also known as $R A R L / R A S L 8 C$, is located in 16p13.3. It is a member of the $R A S$ oncogene family and encodes the protein RAB40C. The RAB family of small GTPases was regards as the cellular regulators of vesicular transport, ${ }^{10}$ and $\mathrm{RAB}$ proteins were the key regulators of eukaryotic biofilm transport in all eukaryotes. ${ }^{11}$ Studies have found that RAB40C was directly regulated by let-7a and played an important regulatory role in the biological role of gastric tumorigenesis. ${ }^{12}$ Aberrant methylation in RAB40C may be related to the pathogenesis of prostate cancer. ${ }^{13}$ Our previous work has proved that RAB40C is a novel lipid droplet-related RAB protein, ${ }^{11}$ which is one of the RAB proteins regulating lipid droplets (LDs) homeostasis. ${ }^{14}$ Lipid metabolism disorder is considered as the main factor of the pathogenesis of alcohol-induced ONFH. ${ }^{15}$ Thus, the occurrence of alcohol-induced ONFH may be relevant to $R A B 40 C$ gene.

In order to verify this hypothesis, this study aimed to search for the association between $R A B 40 C$ single nucleotide polymorphisms (SNPs) and alcohol-induced ONFH susceptibility, so as to provide guidance for the potential treatment and prevention of the disease.

\section{Materials and Methods}

\section{Ethical Statement}

The ethical approval of this study is in line with the ethical principles of the Helsinki declaration on human medical research. Our study has been approved by the ethics committee of Hong Hui hospital in Xi 'an, China, and all participants have signed informed consent before participating in the study.

\section{Study Participants}

All subjects were the Chinese Han population and included 201 patients and 201 controls. Participants underwent routine physical examinations including internal medicine, surgery, and specialized facial examinations. The diagnostic criteria for alcohol-induced ONFH are based on the clinical manifestations of hip, lumbar, and knee pain and mobility limitations. We further diagnosed the disease by MRI analysis and X-ray examination, such as deformities of the femoral head, hip stenosis, protuberances, or collapsed cartilage fractures. ${ }^{16}$ The patients were diagnosed with ONFH after using plain radiographs in stage II, III, and IV of the Ficat Classification systems. Stage I is characterized by no radiological abnormalities. Only some joints are stiff and painful, usually with limited joint movement. The symptoms were relieved after rest, and no positive results were found on X-ray films. Occasionally, uniform or spotty osteoporotic areas could be seen. The second stage is characterized by bone reconstruction on X-ray film, with sparse bone and diffuse bone, but no change in the shape of femoral head or joint space. A plain or CT scan of the femoral head shows osteosclerosis, focal osteoporosis, or cystic changes. Stage III is characterized by continuous fracture of subchondral trabeculae with obvious cystic changes and sclerotic margin around it. The femoral head is flattened due to subchondral fracture, mainly in the load-bearing area. Stage IV is characterized by progressive enlargement of subchondral osteonecrosis, further compression and destruction of the femoral head and acetabulum, with narrowing of joint space and typical changes of osteoarthritis. ${ }^{16}$ The enrolled patients had a history of drinking pure alcohol $>400 \mathrm{~mL}$ $(320 \mathrm{~g} / \mathrm{week}$, any alcoholic beverage) per week for six months or more. The inclusion criteria of the control group were as follows: (1) healthy, excluding asymptomatic ONFH (stage I) subjects; (2) age and BMI-matched Han Population in the control group; (3) no recent infection; (4) no other medical history; (5) no history of alcohol abuse.

\section{SNP Selection and Genotyping}

In 1000 genome project (http://www.internationalgenome. org/), we selected $R A B 40 C$ candidate SNP sites with allele frequency (MAF) over 5\%. Three SNPs (rs4984677, 
rs62030917 and rs2269556) in $R A B 40 C$ were finally identified in the case - control study. We isolated the genomic DNA from the whole blood sample using the Goldmagmini Purification Kit (GoldMag Co. Ltd, Xi'an, China), and DNA concentration was measured using the NanoDrop 2000 (Thermo Scientific, Waltham, MA). Multiplexed SNP Mass EXTEND assay was designed by Agena MassARRAY Assay Design 4.0 software, and SNP genotyping was performed by Agena MassARRAY RS1000 (Agena, San Diego, CA, USA) according to the standard scheme. ${ }^{17}$ We also used Agena Typer 4.0 software to analyze and manage our data. ${ }^{18}$

\section{Data Analyses}

We used Microsoft Excel (Microsoft, Redmond, WA) and SPSS Statistics (version 17.0, SPSS, Chicago, IL) to analyze the collected data. All the $p$-values in the study were two - tailed, and $p<0.05$ was statistically significant. The chi-square test was used to evaluate the deviation of Hardy-Weinberg equilibrium (HWE). Pearson Chi - square test or Fisher's accurate test were also used to compare the allele frequency and genotype frequency of alcoholinduced ONFH patients with that of the control group. The association between polymorphisms in the RAB40C gene and the risk of alcohol-induced ONFH was calculated on the basis of logistic regression analysis. Four models (co-dominant, dominant, recessive, and log-additive) were established using PLINK version 1.07 software to assess the association between each sites and the alcohol-induced ONFH risk. The pairwise linkage disequilibrium (LD), haplotype construction, and genetic association of polymorphism loci were assessed using the Haploview software package (version 4.2).

\section{Results}

This study contained 201 alcohol-induced ONFH patients with an average age of $42.68 \pm 12.875$ years old and 201 healthy controls with an average age of $42.87 \pm 13.270$ years old. The independent sample $t$ test showed that no significant difference was between the case group and the control group. In the case group, there were 54 cases in stage I and II, 147 cases in stage III and IV. Moreover, 44 cases with unilateral lesions and 157 cases with bilateral lesions. The information of BMI, alcohol and tobacco use in both cases and controls were shown in the Table 1. Supplementary Table S1 listed the plasma lipoprotein and lipid levels between patients and controls, and the
Table I Comparison of Clinical Data in Case and Control Groups

\begin{tabular}{|c|c|c|c|}
\hline Characteristic & Case & Control & $p$-value \\
\hline Number & 201 & 201 & \\
\hline Age(years, Mean \pm SD) & $42.68 \pm 12.88$ & $42.87 \pm 13.27$ & $0.888^{\mathrm{a}}$ \\
\hline BMI $\left(\mathrm{kg} / \mathrm{m}^{2}\right.$, Mean $\left.\pm \mathrm{SD}\right)$ & $21.15 \pm 2.70$ & $21.33 \pm 2.87$ & $0.383^{\mathrm{a}}$ \\
\hline $\begin{array}{l}\text { Drinking } \\
\text { Yes } \\
\text { Deletion }\end{array}$ & $\begin{array}{c}199(99 \%) \\
2(1 \%)\end{array}$ & $\begin{array}{c}199(99 \%) \\
2(1 \%)\end{array}$ & $0.688^{b}$ \\
\hline $\begin{array}{l}\text { Smoking } \\
\text { No } \\
\text { Yes }\end{array}$ & $\begin{array}{c}20(10 \%) \\
179(90 \%)\end{array}$ & $\begin{array}{c}25(\mid 2.4 \%) \\
\mid 76(87.6 \%)\end{array}$ & $0.527^{b}$ \\
\hline $\begin{array}{l}\text { Hip lesions } \\
\text { Unilateral } \\
\text { Bilateral }\end{array}$ & $\begin{array}{c}44 \text { (22\%) } \\
\text { I } 57 \text { (78\%) }\end{array}$ & & \\
\hline $\begin{array}{l}\text { Clinical stages } \\
\qquad \begin{array}{l}\text { I+II } \\
\text { III+IV }\end{array}\end{array}$ & $\begin{array}{c}54(27 \%) \\
147(73 \%)\end{array}$ & & \\
\hline
\end{tabular}

Notes: ${ }^{a}$ Independent samples $t$ test; ${ }^{b}$ Pearson Chi-squared test. $p<0.05$ indicates statistical significance.

Abbreviation: SD, standard deviation.

results of the table indicated that there was a significant difference in PLT levels between the two groups.

Supplementary Table S2 showed the primers were used for this study. We have successfully genotyped three SNPs of $R A B 40 C$ gene, and the genotype frequency distribution of all SNPs in the control groups did not deviate from the HWE $(p>0.05)$. Table 2 showed the basic information of chromosome position, role, MAF (Minor allele frequency) of cases and controls and HWE $p$-value of the three SNPs located in $R A B 40 C$ gene. In these three SNPs, the minor allele $\mathrm{G}$ of rs62030917 was significantly associated with an increased alcohol-induced ONFH risk (OR $=1.47,95 \%$ $\mathrm{CI}=1.07-2.02, p=0.017$ ).

Then, we used four genetic models (co-dominant, dominant, recessive, and log-additive models) to analyze the relationship between three SNPs and alcohol-induced ONFH risk (Table 3). The result indicated that carriers with G/A-G/G genotype in rs62030917 were more likely to have alcohol-induced ONFH risk compared with AA homozygous carriers (adjusted $\mathrm{OR}=1.52,95 \% \mathrm{CI}=1.02-2.26, p=0.039$ ) in the dominant model. In the log-additive model, rs62030917 also increased the risk of alcohol-induced ONFH (adjusted OR $=1.42,95 \% \mathrm{CI}=1.05-1.93, p=0.025$ ).

In addition, we also analyzed the association between these loci and the risk of alcoholic osteonecrosis by age stratification and hip lesions stratification (Table 4). Our 
Table 2 Basic Information of the Three SNPs in This Study

\begin{tabular}{|c|c|c|c|c|c|c|c|c|c|c|}
\hline \multirow[t]{2}{*}{ SNP ID } & \multirow[t]{2}{*}{ Gene } & \multirow[t]{2}{*}{ Chr } & \multirow[t]{2}{*}{ Position } & \multirow[t]{2}{*}{ Role } & \multirow[t]{2}{*}{ Alleles A/B } & \multicolumn{2}{|c|}{ MAF } & \multirow[t]{2}{*}{ HWE $p$-value } & \multirow[t]{2}{*}{ OR $(95 \% \mathrm{CI})$} & \multirow[t]{2}{*}{$p^{\mathbf{a}}$} \\
\hline & & & & & & Case & Control & & & \\
\hline Rs4984677 & RAB40C & $16 p \mid 3.3$ & 621,682 & Intronic & $A / G$ & 0.392 & 0.356 & 1.000 & I.17 (0.88-I.56) & 0.290 \\
\hline Rs62030917 & RAB40C & $16 p \mid 3.3$ & 622,040 & Intronic & $\mathrm{G} / \mathrm{A}$ & 0.295 & 0.221 & 0.218 & $1.47(1.07-2.02)$ & $0.017^{*}$ \\
\hline Rs2269556 & RAB40C & $16 p \mid 3.3$ & 625,215 & UTR3 & $\mathrm{G} / \mathrm{A}$ & 0.413 & 0.381 & 0.767 & $1.14(0.86-1.52)$ & 0.356 \\
\hline
\end{tabular}

Notes: ${ }^{\text {P}}$ Pearson Chi-squared test; $* p<0.05$ indicates statistical significance.

Abbreviations: SNP, single nucleotide polymorphism; Chr, chromosome; Alleles A/B, Minor/Major alleles; HWE, Hardy Weinberg equilibrium; MAF, minor allele frequency; OR, odds ratio; $95 \% \mathrm{Cl}, 95 \%$ confidence interval.

Table 3 Association Analysis Between SNPs and Alcohol-Induced ONFH Risk

\begin{tabular}{|c|c|c|c|c|c|c|c|c|}
\hline \multirow[t]{2}{*}{ SNP ID } & \multirow[t]{2}{*}{ Model } & \multirow[t]{2}{*}{ Genotype } & \multirow[t]{2}{*}{ Case } & \multirow[t]{2}{*}{ Control } & \multicolumn{2}{|c|}{ Without Adjusted } & \multicolumn{2}{|c|}{ Adjusted } \\
\hline & & & & & OR (95\% Cl) & $p$ & OR (95\% Cl) & $p$ \\
\hline \multirow[t]{8}{*}{ rs4984677 } & \multirow[t]{3}{*}{ Co-dominant } & G/G & 75 (37.69\%) & 83 (4I.29\%) & I & & I & \\
\hline & & $\mathrm{A} / \mathrm{G}$ & 92 (46.23\%) & $93(46.27 \%)$ & I.IO (0.72-I.67) & 0.676 & I. $10(0.72-1.67)$ & 0.677 \\
\hline & & $\mathrm{A} / \mathrm{A}$ & 32 (I6.08\%) & 25 (I2.44\%) & I. $42(0.77-2.61)$ & 0.263 & I. $42(0.77-2.61)$ & 0.263 \\
\hline & \multirow[t]{2}{*}{ Dominant } & $\mathrm{G} / \mathrm{G}$ & 75 (37.69\%) & $83(4 \mid .29 \%)$ & 1 & & 1 & \\
\hline & & $\mathrm{A} / \mathrm{G}-\mathrm{A} / \mathrm{A}$ & 124 (62.31\%) & II 8 (58.7I\%) & $1.16(0.78-1.74)$ & 0.461 & I.16 (0.78-I.74) & 0.461 \\
\hline & \multirow[t]{2}{*}{ Recessive } & G/G-A/G & 167 (83.92\%) & I 76 (87.56\%) & I & & 1 & \\
\hline & & $\mathrm{A} / \mathrm{A}$ & 32 (16.08\%) & 25 (I2.44\%) & $1.35(0.77-2.37)$ & 0.299 & $1.35(0.77-2.37)$ & 0.299 \\
\hline & Log-additive & - & - & - & I.I7 (0.88-I.55) & 0.293 & I.I7 (0.88-I.55) & 0.293 \\
\hline \multirow[t]{8}{*}{ Rs62030917 } & \multirow[t]{3}{*}{ Co-dominant } & $\mathrm{A} / \mathrm{A}$ & $104(52 \%)$ & 125 (62.19\%) & 1 & & 1 & \\
\hline & & $\mathrm{G} / \mathrm{A}$ & $74(37 \%)$ & $63(31.34 \%)$ & $1.41(0.92-2.16)$ & 0.112 & $1.41(0.92-2.16)$ & 0.112 \\
\hline & & $\mathrm{G} / \mathrm{G}$ & 22 (11\%) & $13(6.47 \%)$ & $2.03(0.98-4.24)$ & 0.058 & $2.04(0.98-4.24)$ & 0.058 \\
\hline & \multirow[t]{2}{*}{ Dominant } & $\mathrm{A} / \mathrm{A}$ & $104(52 \%)$ & $125(62.19 \%)$ & I & & 1 & \\
\hline & & G/A-G/G & $96(48 \%)$ & $76(37.81 \%)$ & $1.52(1.02-2.26)$ & $0.039 *$ & $1.52(1.02-2.26)$ & $0.039 *$ \\
\hline & \multirow[t]{2}{*}{ Recessive } & A/A-G/A & I78(89\%) & 188(93.53\%) & 1 & & 1 & \\
\hline & & $\mathrm{G} / \mathrm{G}$ & $22(11 \%)$ & $13(6.47 \%)$ & I.79 (0.87-3.66) & 0.112 & $1.79(0.87-3.66)$ & 0.112 \\
\hline & Log-additive & - & - & - & $1.42(1.05-1.93)$ & $0.024 *$ & $1.42(1.05-1.93)$ & $0.025 *$ \\
\hline \multirow[t]{8}{*}{ Rs2269556 } & \multirow[t]{3}{*}{ Co-dominant } & $\mathrm{A} / \mathrm{A}$ & $72(36 \%)$ & $78(38.8 \%)$ & I & & 1 & \\
\hline & & $\mathrm{G} / \mathrm{A}$ & $91(45.5 \%)$ & $93(46.27 \%)$ & $1.06(0.69-1.63)$ & 0.791 & I.06 (0.69-I.63) & 0.792 \\
\hline & & $\mathrm{G} / \mathrm{G}$ & $37(18.4 \%)$ & $30(\mid 4.93 \%)$ & I.34 (0.75-2.38) & 0.326 & $1.34(0.75-2.38)$ & 0.327 \\
\hline & \multirow[t]{2}{*}{ Dominant } & $\mathrm{A} / \mathrm{A}$ & $72(36 \%)$ & $78(38.81 \%)$ & I & & I & \\
\hline & & G/A-G/G & $128(64 \%)$ & $123(61.19 \%)$ & $1.13(0.75-1.69)$ & 0.562 & I.I3 (0.75-I.69) & 0.563 \\
\hline & \multirow[t]{2}{*}{ Recessive } & A/A-G/A & $163(81.5 \%)$ & $|7|(85.07 \%)$ & I & & I & \\
\hline & & $\mathrm{G} / \mathrm{G}$ & $37(18.5 \%)$ & $30(\mid 4.93 \%)$ & $1.29(0.76-2.19)$ & 0.338 & $1.29(0.76-2.19)$ & 0.339 \\
\hline & Log-additive & - & - & - & $1.14(0.86-1.50)$ & 0.366 & $1.14(0.86-1.50)$ & 0.367 \\
\hline
\end{tabular}

Notes: $*_{p}<0.05$ indicates statistical significance.

Abbreviations: SNP, single nucleotide polymorphism; OR, odds ratio; $95 \% \mathrm{Cl}, 95 \%$ confidence interval. 


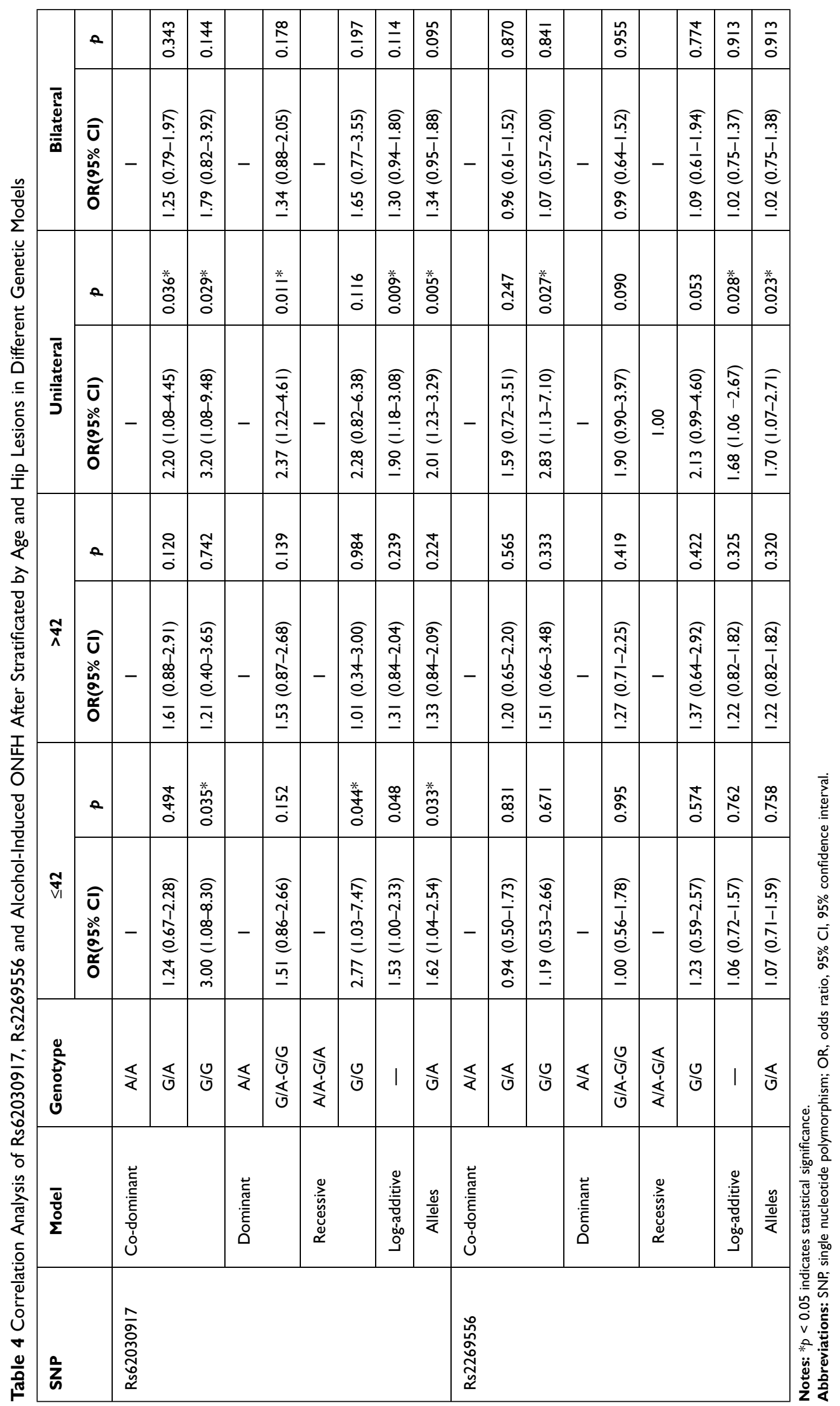


results suggested that rs62030917 increased the risk of alcohol-induced ONFH among people $\leq 42$ years old shown in the allele model $(\mathrm{OR}=1.62,95 \% \mathrm{CI}=1.04$ $2.54, p=0.033)$, -the co-dominant model $(\mathrm{OR}=2.99$, $95 \% \mathrm{CI}=1.08-8.30, p=0.035)$, the recessive model (OR $=2.77,95 \% \mathrm{CI}=1.03-7.47, p=0.044)$ and - the logadditive model $(\mathrm{OR}=1.53,95 \% \mathrm{CI}=1.00-2.33, p=$ 0.048). In patients with unilateral lesions vs controls, rs62030917 conveyed a increasing risk of alcoholinduced ONFH in the allele model (OR $=2.01,95 \% \mathrm{CI}$ $=1.23-3.29, p=0.005)$, the co-dominant model $(\mathrm{OR}=$ $2.20,95 \% \mathrm{CI}=1.08-4.45, p=0.036$; OR $=3.20,95 \% \mathrm{CI}$ $=1.08-9.48, p=0.029)$, the dominant model $(\mathrm{OR}=2.37$, $95 \% \mathrm{CI}=1.22-4.61, p=0.011)$ and the log-additive model $(\mathrm{OR}=1.90,95 \% \mathrm{CI}=1.18-3.08, p=0.009)$. Likewise, rs2269556 also increased the risk of alcoholinduced ONFH (patients with unilateral lesions vs controls $)$ in the allele model $(\mathrm{OR}=1.70,95 \% \mathrm{CI}=1.07-2.71$, $p=0.023)$, the co-dominant model ( $\mathrm{OR}=2.83,95 \% \mathrm{CI}=$ $1.13-7.10, p=0.027)$ and the log-additive model $(\mathrm{OR}=$ $1.68,95 \% \mathrm{CI}=1.06-2.67, p=0.028)$.

Linkage disequilibrium (LD) blocks composed of rs62030917 and rs2269556 were found in unilateral lesions (Figure 1) and older than 42 years old groups (Figure 2), respectively. However, there are no statistically significant correlation between the risk of alcohol-induced ONFH and haplotype. Comparative analysis of plasma

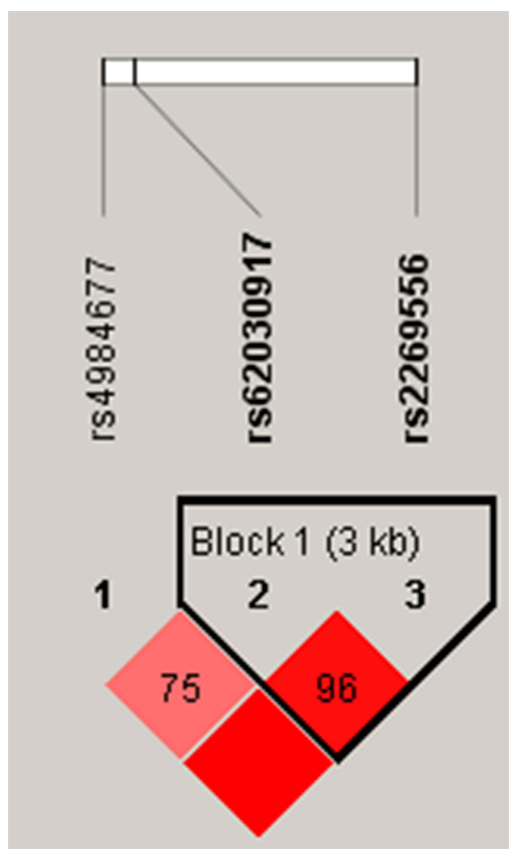

Figure I In patients with unilateral lesions, LD plots containing three SNPs from RAB40C.

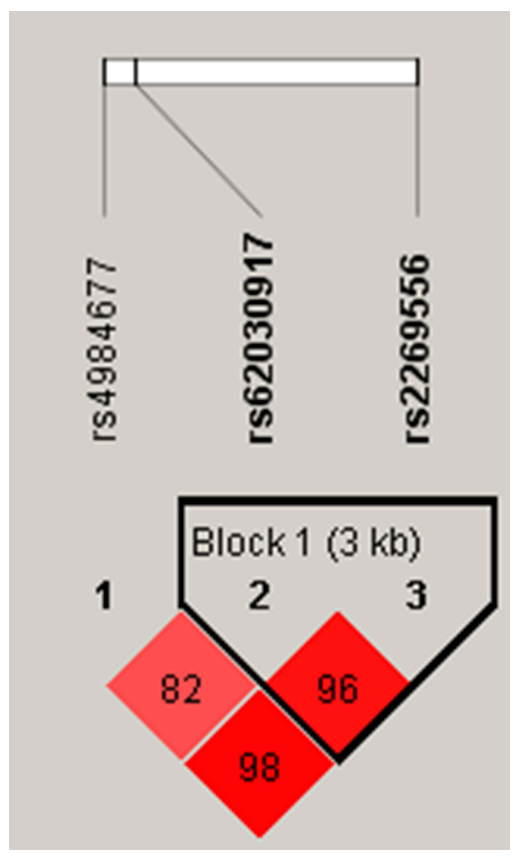

Figure 2 In patients older than 42 years old, LD plots containing three SNPs from RAB40C.

lipoprotein and lipid levels (TC, TG, HDL-C, LDL-C and PLT) in carrier with different genotypes of rs4984677, rs62030917 and rs2269556 was shown in Table 5. The results showed that carriers of AA, GA and GG genotypes in rs2269556 had LDL-C levels of $2.55 \pm$ $0.71 \mathrm{mmol} / \mathrm{L}, 2.78 \pm 0.83 \mathrm{mmol} / \mathrm{L}$ and $2.95 \pm 1.08 \mathrm{mmol} /$ $\mathrm{L}$, respectively, which were significantly different $(p=$ 0.047). Among them, carriers of GG genotype had the highest LDL-C levels.

\section{Discussion}

The pathogenesis of non-traumatic osteonecrosis is vascular injury, osteocyte death, or defective bone repair. ${ }^{19,20}$ Long term excessive drinking can lead to dyslipidemia and then induce ONFH. ${ }^{21,22}$ In recent years, people began to pay attention to the relationship between hereditary susceptibility and alcohol-induced ONFH. $^{23-25}$ Our study on the relationship $R A B 40 C$ gene polymorphisms and alcohol-induced ONFH is the novel study.

The coding region of $R A B 40 C$ contains 281 amino acids, which is longer than most small GTPases because it contains a unique SOCS box domain between the conserved GTPase domain and the pre-acylated C-terminal. Special SOCS box domain interacted with the elongated protein $\mathrm{B} / \mathrm{C}$ and $\mathrm{Cul} 5$ modules, and binded with the ring finger proteins to form active ubiquitin ligases, which 
Table 5 Comparative Analysis of Plasma Lipoprotein and Lipid Levels in Carrier with Different Genotypes of Rs4984677, Rs62030917 and Rs2269556

\begin{tabular}{|c|c|c|c|c|c|c|}
\hline SNP & & $\mathrm{TC}(\mathrm{mmol} / \mathrm{L})$ & TG (mmol/L) & HDL-C (mmol/L) & LDL-C (mmol/L) & PLT $\left(10^{9} / \mathrm{L}\right)$ \\
\hline \multirow[t]{4}{*}{ Rs4984677 } & $\mathrm{AA}(\mathrm{n}=32)$ & $4.70 \pm 1.05$ & $1.69 \pm 0.85$ & $1.03 \pm 0.25$ & $2.91 \pm 1.06$ & $228.53 \pm 71.16$ \\
\hline & $A G(n=92)$ & $4.74 \pm 0.91$ & $1.88 \pm 1.20$ & $1.06 \pm 0.24$ & $2.77 \pm 0.84$ & $229.5 I \pm 53.58$ \\
\hline & $\mathrm{GG}(\mathrm{n}=75)$ & $4.50 \pm 0.86$ & $1.99 \pm 1.53$ & $1.02 \pm 0.22$ & $2.58 \pm 0.7 \mathrm{I}$ & $230.13 \pm 60.63$ \\
\hline & $p$ & 0.244 & 0.551 & 0.366 & 0.120 & 0.964 \\
\hline \multirow[t]{4}{*}{ Rs62030917 } & $A A(n=104)$ & $4.59 \pm 0.89$ & $2.02 \pm 1.42$ & $1.03 \pm 0.23$ & $2.65 \pm 0.84$ & $230.94 \pm 62.13$ \\
\hline & $\mathrm{GA}(\mathrm{n}=74)$ & $4.73 \pm 0.92$ & $1.73 \pm 1.17$ & $1.08 \pm 0.25$ & $2.8 I \pm 0.78$ & $227.58 \pm 53.90$ \\
\hline & $G G(n=22)$ & $4.63 \pm 1.07$ & $1.85 \pm 0.88$ & $0.99 \pm 0.24$ & $2.82 \pm 1.13$ & $232.4 I \pm 75.66$ \\
\hline & $p$ & 0.611 & 0.331 & 0.225 & 0.387 & 0.916 \\
\hline \multirow[t]{4}{*}{ Rs2269556 } & $\mathrm{AA}(\mathrm{n}=72)$ & $4.52 \pm 0.88$ & $2.04 \pm 1.55$ & $1.02 \pm 0.22$ & $2.55 \pm 0.7 \mathrm{I}$ & $228.54 \pm 66.49$ \\
\hline & $\mathrm{GA}(\mathrm{n}=91)$ & $4.72 \pm 0.89$ & $1.81 \pm 1.20$ & $1.07 \pm 0.24$ & $2.78 \pm 0.83$ & $231.65 \pm 52.81$ \\
\hline & $G G(n=37)$ & $4.76 \pm 1.06$ & $1.79 \pm 0.85$ & $1.03 \pm 0.26$ & $2.95 \pm 1.08$ & $227.43 \pm 67.48$ \\
\hline & $p$ & 0.296 & 0.472 & 0.396 & $0.047^{*}$ & 0.918 \\
\hline
\end{tabular}

Notes: ${ }^{*} p<0.05$ indicates statistical significance.

Abbreviations: TC, Total cholesterol; TG, Triglycerides; LDL-C, Low-density lipoprotein-cholesterol; HDL-C, High-density lipoprotein-cholesterol; ApoAI, Apolipoprotein AI; ApoB, Apolipoprotein B; PLT, platelet count.

mediated a series of cellular processes. ${ }^{26-28}$ Our previous work has proved that RAB40C is a novel LDs-related RAB protein. ${ }^{11}$ This is because there is a unique SOCS box domain of RAB40C, which is necessary for LDs cluster. ${ }^{14}$ As the proadipocytes differentiate into adipocytes, the expression of $R A B 40 C$ increased. During the formation and maturation of LDs in adipocytes, RAB40C gradually accumulated to the surface of LDs. RAB40C knockout moderately reduced the size of LDs, suggesting that $\mathrm{RAB} 40 \mathrm{C}$ is involved in the biological genetic process of LDs. ${ }^{29}$

Alcohol induces cell differentiation into adipocytes. ${ }^{30}$ With the increase of alcohol exposure time and concentration, the number of adipocytes was increased. ${ }^{31}$ In the alcohol treatment group, intracellular lipid deposition also occurred, which eventually leaded to the death of osteocytes. ${ }^{31,33}$ These effective findings suggested that alcohol can directly induce adipogenesis, reduce bone marrow mesenchymal osteogenesis, and produce intracellular lipid deposition leading to osteocyte death, which may be related to the occurrence of alcohol-induced ONFH. ${ }^{31}$ In view of the research, rs62030917 of RAB40C significantly increased the risk of alcohol-induced ONFH. In subjects with unilateral lesions, rs62030917 and rs2269556 increased the alcohol-induced ONFH risk. Rs62030917 increased the risk of alcohol-induced ONFH in people $\leq 42$ years old.

To sum up, the expression of $R A B 40 C$ gene increases the risk of alcohol-induced ONFH, which indicates that the gene polymorphisms of $R A B 40 C$ may increase its risk of disease. There are limitations in the scope and quantity of sample selection in this study. In this study, patients and people from Northwest China were selected, which may be biased. In addition, the sample size of this study is small, and further verification of our results needs larger samples to support. Finally, some polymorphic loci with alcohol-induced ONFH have been screened out at the DNA level, and the relationship between polymorphisms and gene expression level has not yet been evaluated. Therefore, the relationship between polymorphisms and gene expression level needs to be evaluated at the RNA level and protein level. The study only provides a direction for alcoholinduced ONFH research.

\section{Conclusion}

Our study indicated that $R A B 40 C$ gene polymorphism rs62030917 significantly increased the risk of alcoholinduced ONFH and it may be a risk locus. Our study 
provides a direction for the research on the mechanism of alcoholic osteonecrosis, but it still needs intensive study.

\section{Funding}

No funds were received in support of this work.

\section{Disclosure}

Chang Liu and Xuan Liu are co-first authors for this study. The authors declare that they have no conflicts of interest.

\section{References}

1. Zalavras CG, Lieberman JR. Osteonecrosis of the femoral head: evaluation and treatment. J Am Acad Orthop Surg. 2014;22 (7):455-464. doi:10.5435/JAAOS-22-07-455

2. Abu-Shakra M, Buskila D, Shoenfeld Y. Osteonecrosis in patients with SLE. Clin Rev Allergy Immunol. 2003;25(1):13-24. doi:10. 1385/CRIAI:25:1:13

3. Yu H, Liu P, Zhu D, et al. Chrysophanic acid shifts the differentiation tendency of BMSCs to prevent alcohol-induced osteonecrosis of the femoral head. Cell Proliferation. 2020;53(8):e12871.

4. Klein RF, Fausti KA, Carlos AS, Ng EL, Tang BL. Ethanol inhibits human osteoblastic cell proliferation. Alcohol Clin Exp Res. 1996;20 (3):572-578. doi:10.1111/j.1530-0277.1996.tb01095.x

5. Guo YJ, Luo SH, Tang MJ, et al. Muscone exerts protective roles on alcohol-induced osteonecrosis of the femoral head. Biomed Pharmacother. 2018;97:825-832. doi:10.1016/j.biopha.2017.11.025

6. Li YZ, Wang Y, Guo YC, et al. OPG and RANKL polymorphisms are associated with alcohol-induced osteonecrosis of the femoral head in the north area of China population in men. Medicine. 2016;95 (25):7.

7. Gaddini GW, Turner RT, Grant KA, Iwaniec UT. Alcohol: a Simple Nutrient with Complex Actions on Bone in the Adult Skeleton. Alcohol Clin Exp Res. 2016;40(4):657-671. doi:10.1111/acer.13000

8. Yoon BH, Jones LC, Chen CH, et al. Etiologic Classification Criteria of ARCO on Femoral Head Osteonecrosis Part 2: alcohol-Associated Osteonecrosis. J Arthroplasty. 2019;34(1):169-174.e161. doi:10.10 16/j.arth.2018.09.006

9. Chen J, Liu W, Cao Y, et al. MMP-3 and MMP-8 single-nucleotide polymorphisms are related to alcohol-induced osteonecrosis of the femoral head in Chinese males. Oncotarget. 2017;8(15): 25177-25188. doi:10.18632/oncotarget.15587

10. Ng EL, Tang BL, Rab GT. Pases and their roles in brain neurons and glia. Brain Res Rev. 2008;58(1):236-246.

11. Tan R, Xu X, Hong W, Wang T. Analysis of biogenesis of lipid droplets by examining Rab40c associating with lipid droplets. Methods Mol Biol. 2015;1270:125-135.

12. Yang Q, Jie Z, Cao H, et al. Low-level expression of let-7a in gastric cancer and its involvement in tumorigenesis by targeting RAB40C. Carcinogenesis. 2011;32(5):713-722. doi:10.1093/carcin/bgr035

13. Geybels MS, Alumkal JJ, Luedeke M, et al. Epigenomic profiling of prostate cancer identifies differentially methylated genes in TMPRSS2:ERG fusion-positive versus fusion-negative tumors. Clin Epigenetics. 2015;7:128. doi:10.1186/s13148-015-0161-6

14. Luo X, Li C, Tan R, et al. A RasGAP, DAB2IP, regulates lipid droplet homeostasis by serving as GAP toward RAB40C. Oncotarget. 2017;8(49):85415-85427. doi:10.18632/oncotarget.19 960

15. Song Y, Du Z, Ren M, et al. Association of gene variants of transcription factors PPARgamma, RUNX2, Osterix genes and COL2A1, IGFBP3 genes with the development of osteonecrosis of the femoral head in Chinese population. Bone. 2017;101:104-112. doi:10.1016/j. bone.2017.05.002
16. Zhao D, Zhang F, Wang B, et al. Guidelines for clinical diagnosis and treatment of osteonecrosis of the femoral head in adults (2019 version). J Orthopaedic Translation. 2020;21:100-110. doi:10.1016/ j.jot.2019.12.004

17. Trembizki E, Smith H, Lahra MM, et al. High-throughput informative single nucleotide polymorphism-based typing of Neisseria gonorrhoeae using the Sequenom MassARRAY iPLEX platform. J Antimicrob Chemother. 2014;69(6):1526-1532. doi:10.1093/jac/dkt544

18. Gabriel S, Ziaugra L, Tabbaa D. SNP genotyping using the Sequenom MassARRAY iPLEX platform. Curr Protocols Human Genetics. 2009;2:12. doi:10.1002/0471142905.hg0212s60

19. Assouline-Dayan Y, Chang C, Greenspan A, Shoenfeld Y, Gershwin ME. Pathogenesis and natural history of osteonecrosis. Semin Arthritis Rheum. 2002;32(2):94-124. doi:10.1053/sarh.2002.33724b

20. Su BZ, Yu Y, Yang H, et al. Nitric oxide of genetic variants is associated with alcohol-induced osteonecrosis risk of the femoral head in a Han population. Int J Clin Exp Pathol. 2016;9 (10):10670-10675.

21. Matsuo K, Hirohata T, Sugioka Y, Ikeda M, Fukuda A. Influence of alcohol intake, cigarette smoking, and occupational status on idiopathic osteonecrosis of the femoral head. Clin Orthop Relat Res. 1988;2(234):115-123.

22. Hirota Y, Hirohata T, Fukuda K, et al. Association of alcohol intake, cigarette smoking, and occupational status with the risk of idiopathic osteonecrosis of the femoral head. Am J Epidemiol. 1993;137 (5):530-538. doi:10.1093/oxfordjournals.aje.a116706

23. Wang Y, Yang X, Shi J, et al. Combination analysis of NOS3, ABCB1 and IL23R polymorphisms with alcohol-induced osteonecrosis of the femoral head risk in Chinese males. Oncotarget. 2017;8 (20):33770-33778. doi:10.18632/oncotarget.16809

24. Wang J, Shi X, Yang H, et al. Association between alcohol-induced osteonecrosis of femoral head and risk variants of MMPS in Han population based on a case-control study. Oncotarget. 2017;8 (38):64490-64498. doi:10.18632/oncotarget. 16380

25. Chen JY, Guo YC, Jin TB, et al. Association of MMPs/TIMPs polymorphism with alcohol-induced osteonecrosis of femoral head in the Chinese Han population. Int J Clin Exp Pathol. 2016;9 (8):8231-8238.

26. Chew EH, Hagen T. Substrate-mediated regulation of cullin neddylation. J Biol Chem. 2007;282(23):17032-17040. doi:10.1074/ jbc.M701153200

27. Kamura T, Sato S, Haque D, et al. The Elongin BC complex interacts with the conserved SOCS-box motif present in members of the SOCS, ras, WD-40 repeat, and ankyrin repeat families. Genes Dev. 1998;12(24):3872-3881. doi:10.1101/gad.12.24.3872

28. Kamura T, Maenaka K, Kotoshiba S, et al. VHL-box and SOCS-box domains determine binding specificity for Cul2-Rbx1 and Cul5-Rbx2 modules of ubiquitin ligases. Genes Dev. 2004;18(24):3055-3065. doi:10.1101/gad.1252404

29. Tan R, Wang W, Wang S, et al. Small GTPase Rab40c associates with lipid droplets and modulates the biogenesis of lipid droplets. PLoS One. 2013;8(4):e63213. doi:10.1371/journal.pone.0063213

30. Yu L, Xu Y, Qu H, Yu Y, Li W, Zhao Y. Decrease of MiR-31 induced by TNF-alpha inhibitor activates SATB2/RUNX2 pathway and promotes osteogenic differentiation in ethanol-induced osteonecrosis. J cel physiol. 2019;234(4):4314-4326. doi:10.1002/jcp.27210

31. Wang Y, Li Y, Mao K, Li J, Cui Q, Wang GJ. Alcohol-induced adipogenesis in bone and marrow: a possible mechanism for osteonecrosis. Clin Orthop Relat Res. 2003;410(410):213-224. doi:10.1097/01.blo.0000063602.67412.83

32. Seamon J, Keller T, Saleh J, Cui Q. The pathogenesis of nontraumatic osteonecrosis. Arthritis. 2012;2012:601763. doi:10.1155/2012/60 1763 


\section{Publish your work in this journal}

The International Journal of General Medicine is an international, peer-reviewed open-access journal that focuses on general and internal medicine, pathogenesis, epidemiology, diagnosis, monitoring and treatment protocols. The journal is characterized by the rapid reporting of reviews, original research and clinical studies

across all disease areas. The manuscript management system is completely online and includes a very quick and fair peer-review system, which is all easy to use. Visit http://www.dovepress.com/ testimonials.php to read real quotes from published authors. 